\title{
A new prototype of a non-ferromagnetic non-invasive femoral extendable prosthesis for skeletally immature patients
}

\author{
Vicatos $\mathrm{G}^{1}$, Ginsberg $\mathrm{S}^{2}$, Parsons $\mathrm{AT}^{3}$ \\ $1 \mathrm{PhD}$, Pr Eng; Associate Professor, Mechanical Engineering Department, University of Cape Town \\ 2 Technical officer, Electrical Engineering Department, University of Cape Town \\ 3 MSc student, Mechanical Engineering Department, University of Cape Town
}

Corresponding author: Dr George Vicatos, Mechanical Engineering Department, University of Cape Town; email: george.vicatos@uct.ac.za; tel: +27 21 6502492; mobile: +27 834243404

\begin{abstract}
Background: Modern extendable endoprostheses allow for non-invasive extension procedures to maintain limb length equality. However, these devices are incompatible with normal diagnostic techniques due to ferromagnetic materials and require physicianfacilitated extensions. The aim, therefore, is to develop an extendable implant, capable of carrying out daily minor extensions comparable to natural growth, as well as permitting monitoring of the surrounding soft tissue site through MRI or CT.

Methods: A biocompatible, non-ferromagnetic prototype device has been constructed for in-vitro testing. The titanium body of the device encloses a piezoelectric motor, a gearbox, a lead screw/telescopic shaft and an electronic circuit board. Testing has been performed to quantify the linear force developed by the device, the electronics' survival and behaviour after gamma radiation, the permeability of water through the seals, the rate of extension and the suitability for MRI and CT imaging. In addition, an external control unit was manufactured, and designed to be programmable by the physician, to control both the amount of daily lengthening and the number of extensions until the next assessment. This device transmits power to the receiver of the extendable device (designed for transcutaneous placement), by means of inductively coupled coils.
\end{abstract}

Results: The prototype device, through its driving mechanism of the piezoelectric motor (a non-ferromagnetic motor), gearbox and a lead screw, produced an extension at a rate of $5 \mu \mathrm{m} / \mathrm{min}$ and generated the required force of $500 \mathrm{~N}$ for limb extension under non-invasive external control. Testing under a strong magnetic field of 3 Tesla, the MRI imaging produced a void in the area where the extendable device was placed, but it did not produce artefacts typical of those observed with ferromagnetic materials. The electronic circuitry, within the prototype, withstood the effects of $25 \mathrm{kGy}$ gamma sterilisation (required for biomedical implants prior to implantation), and the lead cable and inductive coil maintained their flexibility. The device was submerged in water at $37^{\circ} \mathrm{C}$ and $10 \mathrm{kPa}$ pressure for a period of 72 hours and no fluid passed its seals into the motor and gearbox chamber. After concluding the mechanical testing, as well as the MRI and gamma radiation, the external control unit successfully transmitted (via the inductively coupled coils) the signals to the piezoelectric motor, which functioned normally.

Conclusions: The preliminary testing proved that the prototype could be developed into a suitable extendable implant delivering sufficient force for limb lengthening. The external electronic control unit can be programmed by the physician to allow daily extensions of the limb at a rate of $5 \mu \mathrm{m} / \mathrm{min}$. At this rate a maximum daily extension of $30 \mu \mathrm{m}$ will take $6 \mathrm{~min}$. Although MRI testing at 3 Tesla magnetic field strength was inconclusive, but produced no artefacts or scattering, literature suggests that at the recommended strength of 1.5 Tesla, the MRI will produce a sharp and clear image of the device in the patient's limb.

Level of evidence: Level 5

Key words: expandable endoprostheses, piezoelectric motor, limb lengthening, MRI-compatible implants 
Citation: Vicatos G, Ginsberg S, Parsons AT. A new prototype of a non-ferromagnetic non-invasive femoral extendable prosthesis for skeletally immature patients. SA Orthop J 2018;17(4):44-51. http://dx.doi.org/10.17159/2309-8309/2018/v17n4a6

Editor: Prof LC Marais, University of KwaZulu-Natal, Durban

Received: July 2017

Accepted: March 2018

Published: November 2018

Copyright: $\odot 2018$ Vicatos G. This is an open-access article distributed under the terms of the Creative Commons Attribution Licence, which permits unrestricted use, distribution and reproduction in any medium, provided the original author and source are credited.

Funding: This prototype extendable device was designed by the authors and manufactured by various industries in Cape Town. It was financed entirely by the Postgraduate Fund of the University of Cape Town (UCT), and by ATTRI Orthopaedics (Pty) Ltd. UCT has also funded the application and maintenance of the patent with reference: U30536WOEP, Patent No 12759507.2 - 1664 / 2723252.

It is envisaged that the extendable implant would be commercially available by a South African company at an affordable cost to the South African patient.

Conflict of interest: There is no conflict of interest to declare.

\section{Background}

Over 40 years ago, the most effective treatment of bone tumours in the limbs of children was amputation of the affected limb. With the help of biomedical engineering, development of prostheses changed the management of children with bone tumours. Children who were close to skeletal maturity could receive adult-size prostheses as long as the reconstruction of the resected long bone and epiphysis would produce a projected acceptable limb-length discrepancy after the completion of skeletal maturity. This approach necessitated a limb stretching during reconstruction to no more than a maximum of $20 \mathrm{~mm}$. For younger patients, however, this approach was not feasible due to potential neurovascular damage and, as a result, a large variety of extendable endoprostheses have been developed, revised and improved on over the past 30 years to accommodate a gradual limb lengthening over the remaining duration of skeletal growth. The motivation for development has been the improvement of patient quality of life through the reduction in revision surgeries for prosthesis extension, which increases both the risk of infection, ${ }^{1}$ and financial implications. This improvement has resulted in producing minimally invasive and non-invasive extendable implants allowing a lengthening of 6 to $10 \mathrm{~mm}$ per procedure to avoid joint stiffness. ${ }^{2}$ Minimally invasive extendable implants require open surgery, during which the lengthening mechanism of the implant is activated. Although lengthening of these implants avoids major soft tissue dissection, the patient is still subjected to a procedure and risk of infection, twice yearly until completion of growth.

Stanmore Implants Worldwide developed the Mark V JTS, ${ }^{3}$ noninvasive extendable prosthesis in 2006. It has significant advantages over previous designs, particularly the non-invasive nature of the device, allowing for extension procedures to be performed in an out-patient setting. In addition, the endoprosthesis provides controlled and measurable lengthening monitored by continuous $\mathrm{X}$-ray scanning. During extension there is no noticeable feeling of discomfort if the stretching of tissues is kept to small amounts of extension. ${ }^{4}$

The drive unit (external to the patient's body) is a magnetic coil which produces a rotating magnetic field that causes the permanent magnet inside the implant to rotate. ${ }^{5}$ The extension of the patient's limb is carried out in a hospital setting and requires a physician to carry out the extension procedures. This means that in the case of the Mark V, extensions can only be carried out sporadically, every six months. Small, daily extensions are impossible as there is no feedback from the implant that enables the measurement of the exact rotation of the motor.

A device that provides such feedback is that of the Implant Cast, which uses a pulse-driven stepper motor, gearbox and lead screw as the driving mechanism. Counting the pulses to the stepper motor, the rotation is monitored and hence the linear extension can be calculated. It was felt, however, that for both the Stanmore Mark $V$ and the Implant Cast extendable devices, the ferromagnetic influence on MRI and CT imaging would not provide a clear picture of any secondary tumour developing in the nearby bone and soft tissues.

This project aims to develop a prototype extendable endoprosthesis, which would consist of parts that would not interfere with MRI and CT imaging and that would provide feedback, from which small daily extensions could be monitored and measured. With that in mind, the prime objective was to source a nonferromagnetic motor that would drive the extendable mechanism. Such a motor is the piezoelectric motor, which uses electric pulses to activate a number of crystals in a sequential pulsating motion, which in turn is configured (within the motor) to a rotational motion. This motor is triggered by a large but definite number of pulses to complete one rotation, and by counting the number of pulses, the angular position of the motor shaft is monitored. Therefore, via the extension mechanism of the device, the linear elongation is calculated.

\section{Materials and methods}

The design of this prototype extendable endoprosthesis, which is aimed at overcoming some of the shortcomings of currently available devices, requires that all aspects of the device be considered, while maintaining biocompatibility. For this, medical grade titanium and ultra-high molecular weight polyethylene (UHMWPE) were the only materials used for the construction of the prototype.

In the following sections, medical indications, graphical representations and logical suggestions will point to the choice of sizes, materials and combinations of mechanical and electronic elements to attain extension of the endoprosthesis.

\section{Dimensions}

Fundamental to the design of an extendable endoprosthetic femoral replacement are the dimensions of the femur and, more specifically, the resection dimensions that the device intends to replace. The removal of osteosarcoma together with reasonable margins to avoid recurrence, results in an average resection length of $115 \mathrm{~mm}$ (range $80-200 \mathrm{~mm}$ ) either proximally or distally. ${ }^{6}$ As such, the overall dimensions of the device must be limited to an overall length of $<200 \mathrm{~mm}$. Reasonable extension, in this case, is deemed to be of sufficient amount to allow for a significant time span between revision surgeries, limiting the risks and financial 
costs associated with surgery. It is not possible to predict at what age the patient would have to undergo a revision surgery to replace the extendable endoprosthesis and thus it should ideally provide for the maximum possible growth remaining, approximately $70 \mathrm{~mm}$ (10-year-old male distal femur). ${ }^{7,8}$

\section{Loads}

The device is expected to remain and be functional in the patient for at least five years. In this time, the endoprosthesis must resist both static and fatigue failure due to normal loading of the femur, as well as loads due to extension.

The initial soft tissue and muscle loading on the femoral modular endoprosthesis, according to Meswania et al., ${ }^{9}$ varies from $1-578 \mathrm{~N}$, with an average of $135 \mathrm{~N}$. Continued investigation of loading at greater values of extension enabled them to establish a linear relationship between load and extension. The variation in load at $6 \mathrm{~mm}$ extension was significantly larger than that of the initial loading, ranging from 33-1 $513 \mathrm{~N}$, an average of $502 \mathrm{~N}$. Regression analyses applied to the curves yielded an initial load of $122 \mathrm{~N}$ increasing to $516 \mathrm{~N}$ at $6 \mathrm{~mm}$. Small extensions require significantly lower distraction forces, further improved by tissue relaxation between extensions. Therefore, it would be an advantage for the design of the prototype to provide elongations at a rate comparable to natural growth, achieving minor extensions frequently in contrast to large periodic extensions. Although the device is intended to carry out frequent minor extensions, should the need arise, it should be capable of sustaining large loads (average $500 \mathrm{~N}$ ), equivalent to those required for a $6 \mathrm{~mm}$ periodic extension.

Of all the long bones, the femur experiences the greatest functional loading, and this is most relevant to the design capabilities of an extendable endoprosthetic replacement. Designed for femoral loads (multiples of body weight, BW), the device would experience an axial force of $3.09 \mathrm{BW},{ }^{9}$ as well as a net moment of 11.5 BWcm. Ascending stairs similarly causes femoral load peaks in the torsional moment of $1.3 \mathrm{BWcm}$. Based on this loading, together with the dimensional constraints on the device, the stresses were calculated using formulae pertinent to engineering applications, as described by Taylor et al. ${ }^{10}$

The overall device exhibits a minimum safety factor of 1.6 based on the fatigue strength of medical grade titanium (approx. $350 \mathrm{MPa})$.

\section{Extension mechanism system}

A lead screw is a simple device, which converts rotation to linear motion, producing elongations.

Equations by Shigley et al..11,12 linking the required axial force of $500 \mathrm{~N}$, possible lead screw diameters, screw-thread pitch and friction forces, indicated that metric threads of the lead screw, of sizes M6, M8, M10 and M12, could be considered in the design of the extension mechanism. ( $M$ designates the metric screw-thread, the numeral indicates the diameter in $\mathrm{mm}$.) All these metric threads can generate the required extension force with an input torque $<2.5 \mathrm{Nm}$ (Figure 1). The correlation of the axial force versus the driving torque shows that the M6 thread is the most suitable, offering the greatest mechanical advantage as well as the smallest diameter. But, in addition to extending the endoprosthesis, the threaded drive-shaft must be sufficiently sized to avoid the possibility of fatigue under normal activities.

The M $8 \times 1$ thread (metric screw thread $8 \mathrm{~mm}$ in diameter and $1 \mathrm{~mm}$ pitch) is the most suitable for the extendable endoprosthesis, providing sufficient mechanical advantage for extension and withstanding the maximum expected axial load without fatigue, based on reasonable activity (participation in active sports is rare

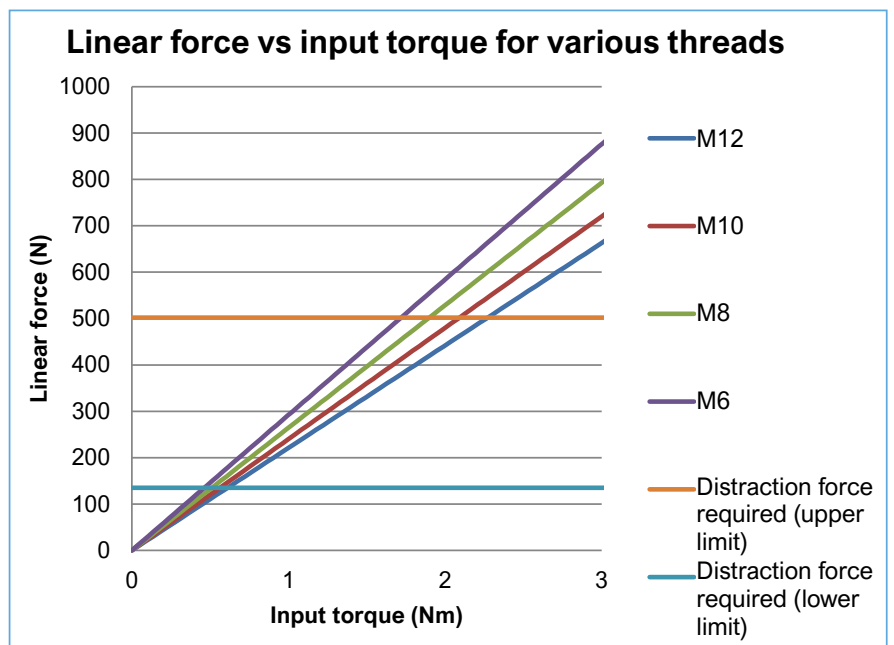

Figure 1. The four lead screws under consideration can produce a force of $500 \mathrm{~N}$, receiving a torque below $2.5 \mathrm{Nm}$

\section{Fatigue considerations of various lead screws}

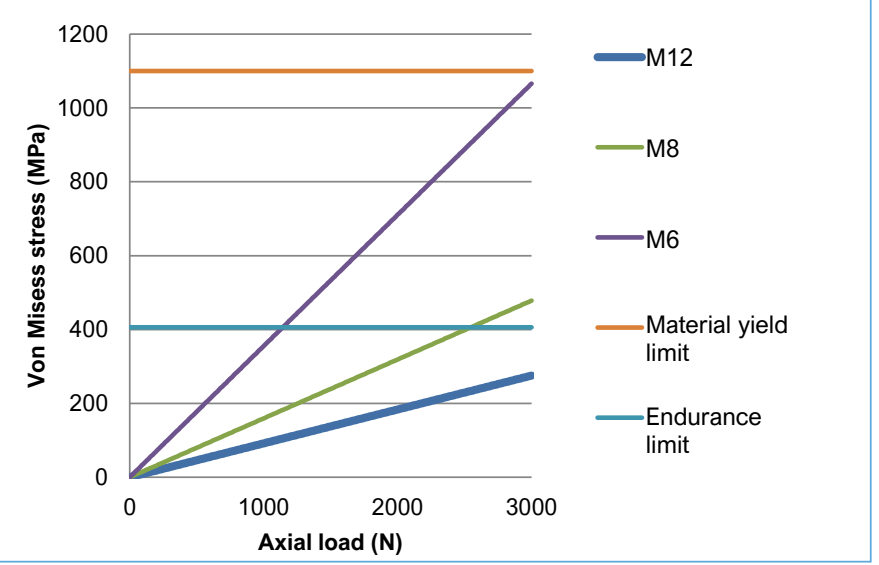

Figure 2. The $6 \mathrm{~mm}$ diameter lead screw, M6, at $2400 \mathrm{~N}$ axial load, will be subjected to stresses above the endurance limit and would fail from fatigue loading

for patients with endoprosthetic replacements). ${ }^{13}$ For example, a person of body mass $70 \mathrm{~kg}$, performing an activity which would result in a force $3.5 \mathrm{BW}$, roughly $2400 \mathrm{~N}$, the M6×1 lead screw would fail due to fatigue (Figure 2), while the M8×1 lead screw is below the endurance limit. Therefore, the $M 8 \times 1$ has become the choice of lead screw for this design.

\section{Drive unit system}

The drive unit is housed within the drive-unit casing of the implant (Figures 3 and 4) and is designed to develop the necessary torque required to overcome the distraction loads of soft tissues.

The recent development in extendable endoprosthesis devices is that the extensions are carried out non-invasively. This necessitates the use of an electrical or magnetic drive system, both of which are employed in the available non-invasive devices. The nature of operation of these drives is that they require ferromagnetic material, which is detrimental to imaging techniques (crucial to the accurate diagnosis of cancer), such as MRI. Recently developed rotary piezoelectric motors overcome the issues associated with MRI. These motors can develop high torques, while containing no ferromagnetic material. They operate by sequentially activating radially spaced piezoelectric crystals, in contrast to the magnetic field interaction of more commonly used stepper motors. 


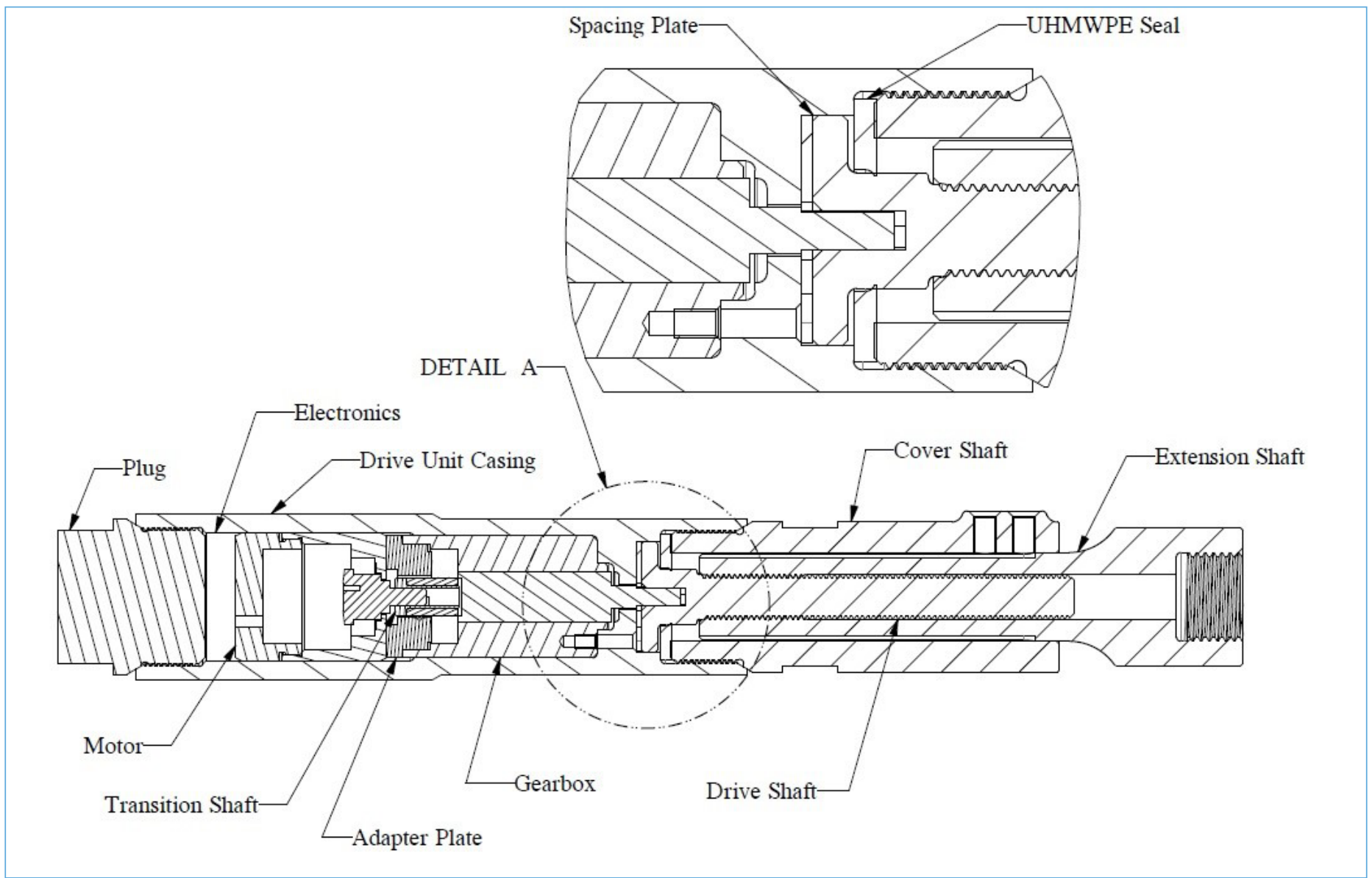

Figure 3. Schematic cut-out showing the drive unit casing and extension mechanism of the prototype test device

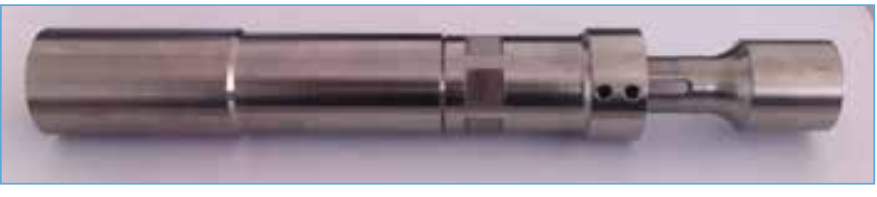

Figure 4. The overall appearance of the titanium prototype test mechanism

The Piezo Legs R01S-12 motor (manufactured by Piezo Motor $\mathrm{Gmbh}$ ) is the most suitable for application in this prototype design, generating a peak output torque of $0.08 \mathrm{Nm}$. This peak torque of the piezo motor cannot be sustained for prolonged operation, and its rated torque of $0.04 \mathrm{Nm}$ alone is insufficient or marginal for developing the necessary axial force required for extension of the mechanism. For this reason, the motor is coupled with a three-stage planetary gearbox, rated at a maximum torque of $3 \mathrm{Nm}$ (manufactured by Wittenstein Cybermotor Gmbh), and with a 196:1 reduction ratio. The combination of the piezo motor, the planetary gearbox and the $M 8 \times 1$ lead screw, can yield a linear force of $800 \mathrm{~N}$ through the extension mechanism, at an extension rate of $5 \mu \mathrm{m}$ per min. This would allow the device to cater for daily extensions comparable to that of natural growth. For example, for female children of 10 years of age, a $30 \mu \mathrm{m}$ daily extension would take $6 \mathrm{~min}^{8}$ (Figure 5).

\section{Power, control and feedlback ${ }^{14}$}

The electronic system is required to:

- Couple the motor drive energy through the patient's skin

- Generate step signals suitable for motor operation

- Monitor and count motor rotation

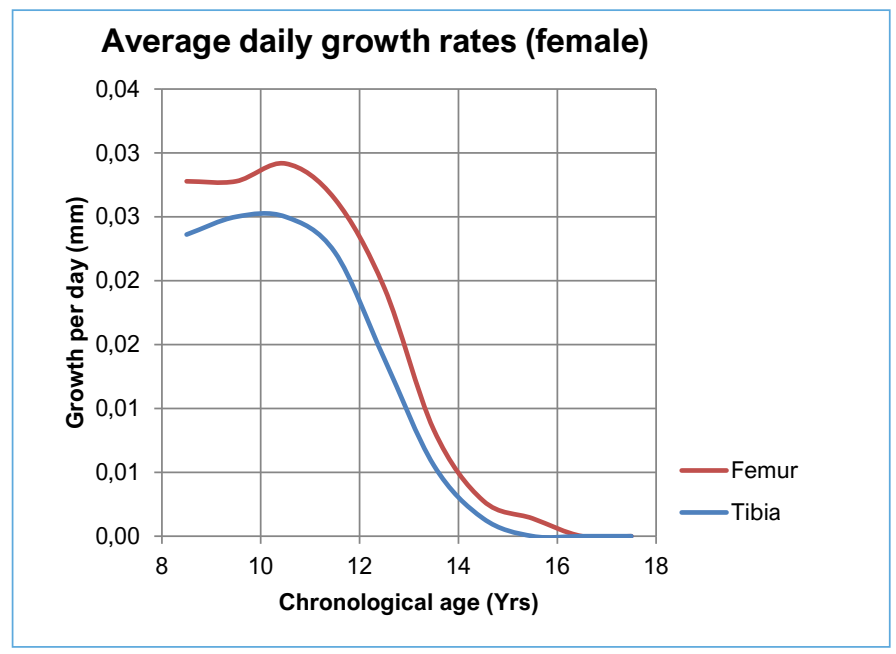

Figure 5. Comparison of female daily growth rates ${ }^{8}$

- Permit programming by medical personnel

- Withstand conditions associated with standard medical procedures including sterilisation and diagnostics.

This electronic system can be separated into three distinct categories: internal, external, and program (allowing configuration).

\section{Internal}

Contained within the extendable prototype device are: motor, motor-drive circuit, power-receiving coil and regulation circuit. The power transmitted is supplied through inductance from a coil outside of the patient's body across the skin. 
To monitor the motor rotation, a pulse feedback from the motordrive circuit causes a similar signal to be generated at the external control unit. The circuitry is so designed that for every four pulses transferred to the motor, a signal in the form of a current impulse (emitted from the motor circuit and received by the external control box circuit), indicates the completion of four steps taken by the motor (that is, 1 pulse $=1$ step). By counting these signals and knowing the number of steps required for the motor to turn one revolution, the exact angular position of the motor shaft is calculated. Following this current impulse, the process is repeated for the next four pulses. The drive frequency is selected to be in the $100 \mathrm{kHz}$ to $1 \mathrm{MHz}$ range, thus avoiding the ionising and thermal range of frequencies that would be harmful to the patient, while still being of high enough frequency to couple effectively the external and internal coils.

\section{External}

The external electronics are housed in a casing designed to be strapped onto the patient, providing a snug fit with the limb. This close contact with the skin ensures the supply of an alternating magnetic field to the internal inductive coil (charge coil, the inductive coil under the patient's skin which powers the motor) below the skin. The circuitry within this external unit monitors the internally generated current impulse, thus measuring the number of steps taken by the motor. The use of a microcontroller within the external unit allows for the device to be interfaced with a computer, typically through a USB port. Various parameters can then be transferred to the unit and stored in memory, such as the total extension required over a given period, as well as limits on daily extensions. Each extension operation results in a countdown on the total extension programmed by the physician. These values (total and daily extensions) are indicated to the user on a liquid crystal display (LCD).

Light emitting diodes (LEDs), present on the face of the case, indicate coupling between the external drive coil and internal charge coil, failing which, extension is not permitted. A single button on the unit allows the patient to begin an extension operation within the limits of the pre-programmed parameters. If coupling is lost, the unit stores the amount of extension carried out and updates the remaining for the programmed period. The flow chart (Figure 6), depicts the logic of the circuitry. A uniquely numbered radio frequency identification (RFID) tag may be implanted in the patient as well. This would allow a reading device in the control unit to read the tag and identify that it is coupled with the correct implant, thus removing the possibility of the incorrect control unit being used on a patient.

\section{Program}

The external control unit can be programmed through a computerbased application. This application will enable the medical practitioner to program the external control unit by entering the amount of extension required and the time period through which the extension will be performed. Having set extension parameters, the settings could be transferred from the computer to the external control unit via a USB connection, or similar.

\section{Sealing against fluids}

The motor and gearbox provided by Piezo Motor and Wittenstein respectively, together with the custom-drive electronics, contain biomedically incompatible materials and as such, cannot be in contact with body tissue or fluid. Effective sealing of the internal components presents difficulties to the sterilisation process, preventing the permeation of sterilising gases involved with autoclaving and ethylene oxide (EtO). Thus, the only effective means of sterilisation is through exposure to gamma radiation.

\section{Sterilisation by gamma radiation}

The need to withstand $25 \mathrm{kGy}$ gamma ionising radiation means that conventional microcontroller technology cannot be used. High dosage radiation causes displacement in the lattice structure of semiconductor devices. This typically causes destruction of circuitry built with CMOS (complementary metal oxide semiconductor) technology, the dominant technology used in the fabrication of microcontroller devices. In addition, both Flash and EEPROM (electrically erasable programmable read only memory) types suffer from degradation and spurious changes to their contents when exposed to large doses of ionising radiation. This effectively rules out the use of any of the standard computational devices in use. To work around these problems the CMOS devices were replaced with bipolar transistors and these were hard-wired to determine the operation of the circuitry. The hard-wiring eliminates the need for any memory devices. While bipolar transistors do suffer degradation due to ionising radiation, this practically results in a shift in operating parameters rather than complete destruction of the device. Robust engineering techniques were used to compensate for the shift in parameters, while still producing the desired operation. Additionally, the circuit components for internal placement must be as small as possible.

\section{Experimental method}

Tests were performed on the prototype to ascertain the transition of power from the control box to the motor before and after gamma irradiation, the ability to produce the required linear force, the sealing capability against fluid accessing the motor chamber, and the compatibility of the prototype device with MRI imaging.

\section{Linear force experiment}

The prototype extendable device was securely fixed on the test bench and linked onto the piston of a fixed calibrated hydraulic cylinder. Extensions of the device were recorded as a movement of the piston of the hydraulic cylinder against a constant load of $490 \mathrm{~N}$ (equivalent to a $50 \mathrm{~kg}$ mass).

\section{Inductive power transfer}

The driving circuit provided by Piezo Motor SE generates a sinusoidal output for driving the motor's piezo crystals. Changing the drive signal from sinusoidal to square waves (pulses) resulted in significant space saving on the circuit board, which was required due to the limited space available inside the implant. It was therefore necessary to establish whether a square wave generated in the control box and transmitted to the subcutaneous coil through the soft tissues could drive the motor. To do this, the receiving and transmitting coils were placed on either side of a section of bovine muscle tissue, the thickness of which was gradually reduced until power transfer was achieved.

\section{Sealing against fluids}

Apart from the drive lead screw and drive shaft, all other parts are contained in a sealed casing provided by tapered metal-on-metal contact. A seal made of UHMWPE is used to seal the drive shaft (lead screw). Sealing at this point is achieved by an interference tolerance-fit between the drive shaft and the seal surface so that it would still allow rotation of the drive shaft. 


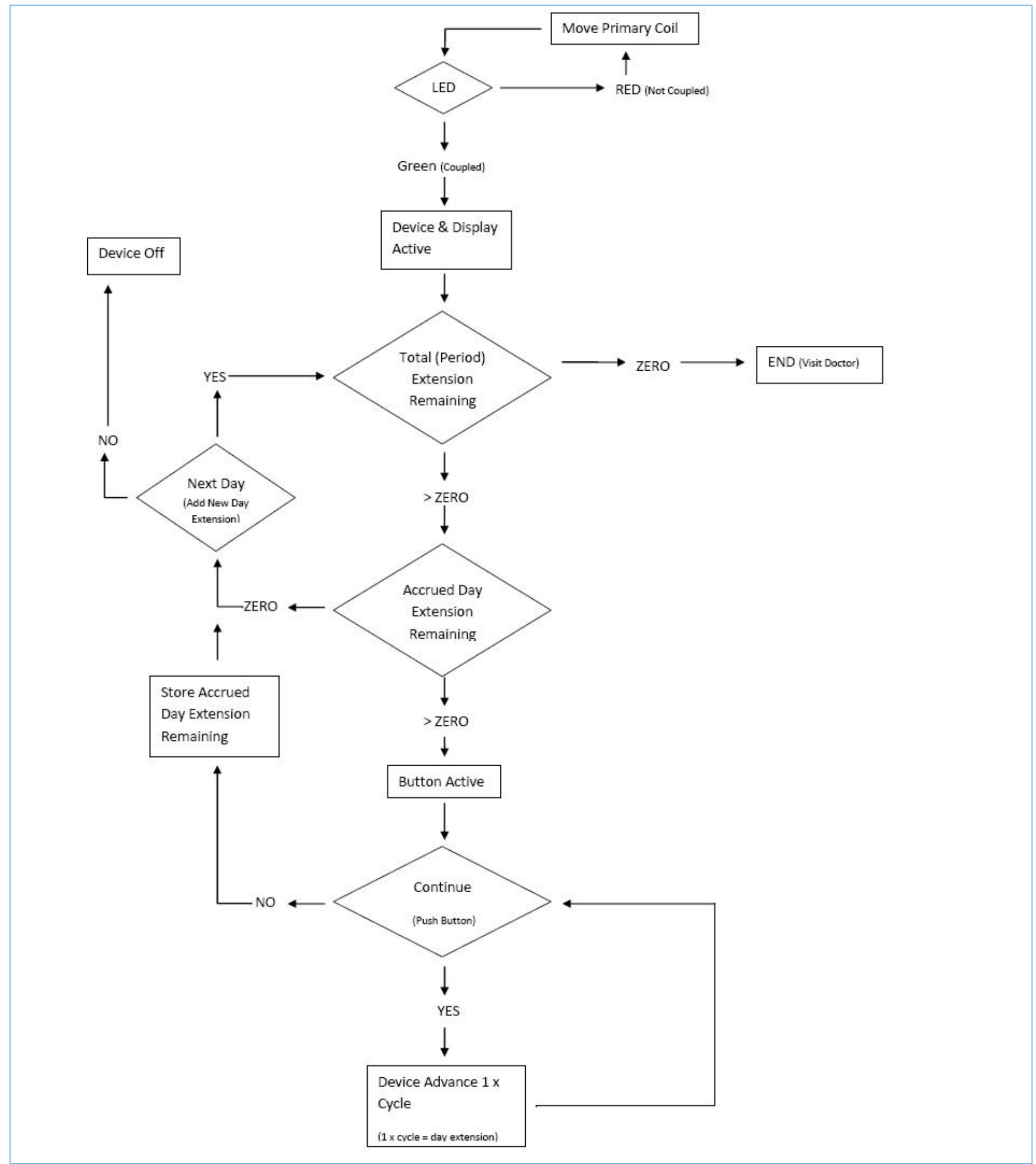

Figure 6. Flow diagram of device control logic

The device was submerged in water at $37^{\circ} \mathrm{C}$ and $10 \mathrm{kPa}$ pressure for a period of 72 hours and periodically the drive shaft was rotated against the UHMWPE seal.

This experiment was completed after sterilisation with gamma irradiation to establish whether a possible change of properties due to cross-linking of the UHMWPE material would affect the sealing performance.

\section{MRI testing}

A piezo motor was chosen for the drive unit to develop an endoprosthetic extension module compatible with MRI and CT imaging. To test this compatibility, the extension module was covered by soft bovine tissue and subjected to a 3 Tesla magnetic strength MRI (the only one available at the time of testing). 


\section{Results}

\section{Linear force}

The device generated a linear force of $490 \mathrm{~N}$ (equivalent to moving a $50 \mathrm{~kg}$ mass), sufficient to develop an extension of $6 \mathrm{~mm}$. This accommodated both a simulation of regular daily extension, as well as periodic extension, similar to that of the Stanmore Mark V. However, it was also noticed that the RPM of the piezo motor decreased linearly with respect to experiment time. This characteristic of the motor was further investigated to rule out the effects of the linear force output and it was confirmed that the RPM was not influenced by the loading, but decreased naturally over time, settling to a constant value.

Subsequent repeated experiments confirmed the device's capability of generating the force of $490 \mathrm{~N}$, which was maintained constant for about $10 \mathrm{~mm}$ of extension.

\section{Seal frictional torque}

In addition to frictional losses in the thread of the drive shaft, there was an opposing torque generated due to friction between the UHMWPE seal and the drive shaft (lead screw). This torque was found to be between 0.028 and $0.036 \mathrm{Nm}$, and as such, it had negligible influence on the linear output force of the extension mechanism.

\section{Inductive power transfer}

Power transfer was achieved through approximately $10 \mathrm{~mm}$ thickness of soft tissue. The drive frequency was maintained at $130 \mathrm{kHz}$, producing no thermal effects on the muscle tissue.

\section{Gamma sterilisation testing}

After exposure of all the components of the device to $25 \mathrm{kGy}$ gamma radiation, all the electronic circuitry (Operational Amplifiers, op-amps [MC33078]) was operational, but required more power than before the exposure to radiation. The power circuitry was able to adjust to this loss of power and the piezo motor functioned normally.

\section{Sealing against fluids}

The UHMWPE seal on the lead screw was tested and shown to prevent the passage of water to the compartment of the device containing other parts of the mechanism, which are of biologically incompatible materials. It was confirmed that for the conditions of the testing, even under the periodic rotation of the drive shaft, no fluid had passed the UHMWPE seal, or the tapered metal-on-metal seals of the implant casing.

\section{MRI testing}

A void in the resulting image, corresponding to the location of the device (Figure 7), resulted in non-conclusive results at 3 Tesla strength magnetic field. However, according to Shellock, ${ }^{15}$ if a metallic object displays weak artefacts at a 1.5 Tesla MRI system, it will exhibit substantial magnetic field interactions during exposure to a 3 Tesla MRI system.

\section{Discussion}

The prime aim of this project was to design and develop a prototype extendable endoprosthesis device that would be compatible with

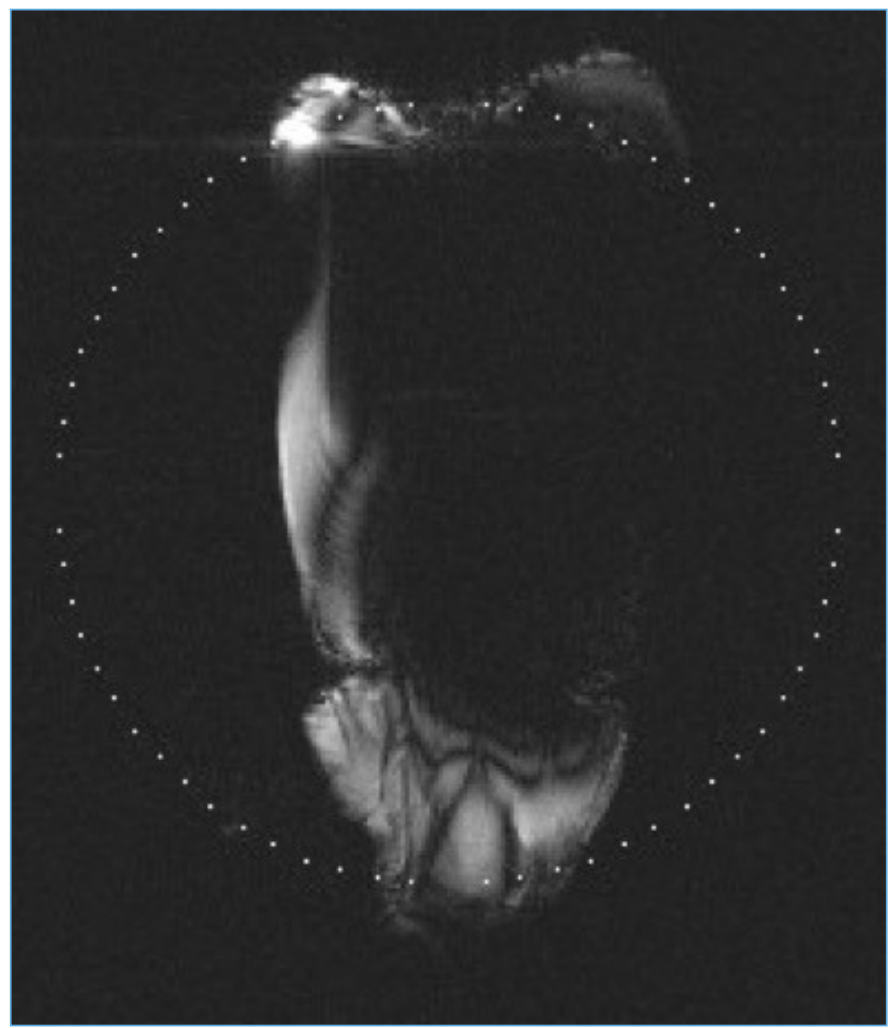

Figure 7. A 3 Tesla magnetic field MRI scan of the prototype expendable endoprosthesis replacing the tibia of bovine bone. Due to high intensity of the magnetic field, the image shows a void instead of an outline of the titanium device, but no metal scatter or artefacts are emitted

MRI and CT imaging, and for this, a piezoelectric motor was used. Additional benefits in using such a motor were that its fine rotational control would provide feedback from which small daily extensions could be assessed. The prototype was built, tested at laboratory conditions, and passed mechanical, electronic and sterilisation processes, and produced sufficient force similar in magnitude to the force experienced in limb-lengthening procedures.

However, there is still an undefined MRI/CT compatibility due to the strength of the MRI's magnetic field used, although there was no image scattering, or artefacts visible. With the assumption that other existing and currently available extendable endoprosthesis devices are not MRI/CT compatible, the proposed prototype compares favourably in function, as it produces a similar extension force.

This device has been designed specifically to produce small, but measurable daily extensions (similar to natural growth), that could be monitored via a programmable interface and as such, it is assumed that the extension force required to overcome tissue tension could be much smaller. Although this force could not be assessed during in-vitro testing, it could be approximated, as there is a linear relationship between tissue tension and elongation.

The overall length of the prototype device is governed by the resection length. However, there is a portion of the device, about $110 \mathrm{~mm}$, that houses the driving electronic circuitry, the motor and the gearbox, which cannot be reduced in length; for example, this prototype device has an overall length of $190 \mathrm{~mm}$ and can provide an $80 \mathrm{~mm}$ extension.

The drive unit inside the implant has been designed to be powered and controlled using inductive coupling across the patient's skin. In-vitro testing (inside tibial bovine tissue) confirmed the efficacy of the electronic controls. A pulse was sent back from the motor after completing four phase pulses, activating the piezo crystals and turning the motor $4 \mu \mathrm{rad}$. In this way, the amount of extension achieved was monitored. 
Table I: Summary of test device specifications

\begin{tabular}{|c|c|c|c|}
\hline \multirow[t]{2}{*}{ Category } & \multicolumn{3}{|c|}{ Details } \\
\hline & Specification & Value & Units \\
\hline \multirow{2}{*}{ Dimensions } & Diameter & 28.1 & $\mathrm{~mm}$ \\
\hline & Length & 190 & $\mathrm{~mm}$ \\
\hline Distraction load & Linear force (as tested) & 500 & $\mathrm{~N}$ \\
\hline \multirow{4}{*}{ Functional load } & Axial & 3.09 & BW \\
\hline & Antero-posterior moment & 9.5 & $\mathrm{BWcm}$ \\
\hline & Medio-lateral moment & 6.7 & BWcm \\
\hline & Torsion & 1.3 & $\mathrm{BWcm}$ \\
\hline \multirow{4}{*}{ Material } & Endoprosthetic & Ti6Al4V & - \\
\hline & Seals & UHMWPE & - \\
\hline & Motor & As supplied & \\
\hline & Gearbox & As supplied & \\
\hline Operation & $\begin{array}{l}\text { Non-invasive, wireless } \\
\text { power and control }\end{array}$ & - & - \\
\hline Extension & Possible extension & 80 & $\mathrm{~mm}$ \\
\hline $\begin{array}{l}\text { Ingress } \\
\text { protection }\end{array}$ & IP & 68 & \\
\hline
\end{tabular}

The external control unit was designed with the possibility that the prototype device could perform extensions similar to those of natural growth and that these daily extension procedures could be carried out at the patient's home, with the physician's permission. Testing of the prototype showed that this could be possible.

Failure to produce a clear MRI image (which would have shown a sharp outline of the device inside the soft tissue), indicates that this particular statement of the aim of the project is still pending; at the time of submitting this paper, there was no funding available for further testing with an MRI of 1.5 Tesla intensity magnetic field.

It is therefore envisaged that with the availability of funds, an assessment with a 1.5 Tesla intensity MRI may secure the efficacy of the device. In addition, further research during in-vivo testing would provide a soft-tissue/force-related feedback to the physician, during daily micrometric extensions. This would entail minor modification of the electronics monitoring the device that would allow signals from the implant to be transmitted to the control box during the daily extensions and stored into a memory circuit, which could then be assessed by the physician.

\section{Conclusions}

Based on the review of both past and currently available devices, areas for improvement were noted and implemented, specifically relating to frequency of extensions and MRI compatibility. Crucial design requirements led to the prototype being subjected to invitro testing, specifically to linear force, mechanical strength, sterilisation compatibility, seal testing, wireless power transfer and device control; all tests were successful (Table I).

MRI compatibility could not be verified. However, because no artefacts or scattering were seen emerging from the MRI image at 3 Tesla magnetic strength, literature indicates, ${ }^{15}$ that the device may be MRI compatible at the standard 1.5 Tesla, which will be confirmed in the future under in-vivo testing. Otherwise, the prototype holds promise as an alternative to available devices.

\section{Ethics statement}

The prototype extendable device, once developed, was tested in vitro, undergoing mechanical strength and endurance validation, as well as control and response of the electronics circuitry. There was therefore no need to obtain ethics approval.

\section{References}

1. Unwin P, Walker P. Extendible endoprostheses for the skeletally immature. Clinical Orthopaedics and Related Research 1996;322:179-93.

2. Neel MD, Wilkins RM, Rao BN, Kelly CM. Early multicenter experience with a non-invasive expandable prosthesis. Clin Orthop Relat Res 2003;415:72-81. doi:10.1097/01. blo.0000093899.12372.25

3. Gupta A, et al. Non-invasive distal femoral expandable endoprosthetic for limb-salvage surgery in paediatric tumours. Journal of Bone and Joint Surgery 2006;88(5):649-54.

4. Stanmore Implants, W., n.d. JTS Drive Unit: Operations Manual.

5. Gupta A, et al. Stanmore non-invasive growing arthrodesis endoprosthetic in the reconstruction of complicated total knee arthroplasty. A case report. The Knee 2006;13(3):247-51.

6. Kapukaya A, et al. Limb reconstruction with the callus distraction method after bone tumour resection. Archive of Orthopaedic and Trauma Surgery 2000;120:215-18.

7. Anderson M, Green WT, Messner MB. Growth and prediction of growth in the lower extremities. Journal of Bone and Joint Surgery 1963;45:1-14.

8. Anderson M, Green WT. Lengths of the femur and tibia. American Journal of Disease of Children 1948;75:279-90.

9. Meswania JM, Walker PS, Sneath RS, Grimer RJ. In vivo distraction forces in extendible endoprostheticc replacements - a study of 34 patients. Proceedings of the Institution of Mechanical Engineers 1998;212(H):151-55.

10. Taylor S, Walker P. Forces and moments telemetered from two distal femoral replacements during various activities. Journal of Biomechanics 2001;34:839-48.

11. Shigley JE, Mischke CR, Budynas RG, n.d. Load and Stress Analysis. Mechanical Engineering and Design. 7th Edition ed. s.I.:s.n.

12. Shigley JE, Mischke CR, Budynas RG, n.d. The Mechanics of Power Screws. Mechanical Engineering Design. 7th Edition ed. s.l.:s.n., p. 400-408.

13. Grimer RJ. Surgical options for children with osteosarcoma. Lancet Oncology. 2005;6:85-92.

14. Vicatos G, Ginsberg S, Parsons A. European patent No. 12759507.2-1664/12759507.2, Reference U30536WOEP

15. Shellock FG. Reference manual for MR safety, implants, and devices, 2008. Los Angeles: Biomedical Publishing Group. 